\title{
Monotone and convex positive solutions for fourth-order multi-point boundary value problems
}

Yang Liu ${ }^{1,2^{*}}$, Zhang Weiguo ${ }^{1}$ and Shen Chunfang ${ }^{2}$

\author{
* Correspondence: yliu1219@163. \\ com \\ ${ }^{1}$ College of Science, University of \\ Shanghai for Science and \\ Technology, Shanghai 200093, PR \\ China \\ Full list of author information is \\ available at the end of the article
}

\begin{abstract}
The existence results of multiple monotone and convex positive solutions for some fourth-order multi-point boundary value problems are established. The nonlinearities in the problems studied depend on all order derivatives. The analysis relies on a fixed point theorem in a cone. The explicit expressions and properties of associated Green's functions are also given.
\end{abstract}

MSC: 34B10; 34B15.

Keywords: multi-point boundary value problem, positive solution, cone, fixed point

\section{Introduction}

Boundary value problems for second and higher order nonlinear differential equations play a very important role in both theory and applications. For example, the deformations of an elastic beam in the equilibrium state can be described as a boundary value problem of some fourth-order differential equations. Owing to its importance in application, the existence of positive solutions for nonlinear second and higher order boundary value problems has been studied by many authors. We refer to recent contributions of Ma [1-3], He and Ge [4], Guo and Ge [5], Avery et al. [6,7], Henderson [8], Eloe and Henderson [9], Yang et al. [10], Webb and Infante [11,12], and Agarwal and O'Regan [13]. For survey of known results and additional references, we refer the reader to the monographs by Agarwal [14] and Agarwal et al. [15].

When it comes to positive solutions for nonlinear fourth-order ordinary differential equations, two point boundary value problems are studied extensively, see [16-24]. Few papers deal with the multi-point cases. Furthermore, for nonlinear fourth-order equations, only the situation that the nonlinear term does not depend on the first, second and third order derivatives are considered, see [16-23]. Few paper deals with the situation that lower order derivatives are involved in the nonlinear term explicitly. In fact, the derivatives are of great importance in the problem in some cases. For example, in the linear elastic beam equation (Euler-Bernoulli equation)

$$
\left(\mathrm{EI} u^{\prime \prime}(t)\right)^{\prime \prime}=f(t), t \in(0, L),
$$

where $u(t)$ is the deformation function, $L$ is the length of the beam, $f(t)$ is the load density, $E$ is the Young's modulus of elasticity and $I$ is the moment of inertia of the cross-section of the beam. In this problem, the physical meaning of the derivatives of the function $u(t)$ is as follows: $u^{(4)}(t)$ is the load density stiffness, $u^{\prime \prime \prime}(t)$ is the shear

(c) 2011 Liu et al; licensee Springer. This is an Open Access article distributed under the terms of the Creative Commons Attribution License (http://creativecommons.org/licenses/by/2.0), which permits unrestricted use, distribution, and reproduction in any medium, provided the original work is properly cited. 
force stiffness, $u^{\prime \prime}(t)$ is the bending moment stiffness and the $u^{\prime}(t)$ is the slope. If the payload depends on the shear force stiffness, bending moment stiffness or the slope, the derivatives of the unknown function are involved in the nonlinear term explicitly.

In this paper, we are interested in the positive solution for fourth-order nonlinear differential equation

$$
x^{(4)}(t)=f\left(t, x(t), x^{\prime}(t), x^{\prime \prime}(t), x^{\prime \prime \prime}(t)\right), t \in[0,1],
$$

subject to multi-point boundary condition

$$
x^{\prime \prime \prime}(1)=0, x^{\prime \prime}(1)=0, x^{\prime}(0)=0, x(1)=\sum_{i=1}^{m-2} \beta_{i} x\left(\xi_{i}\right)
$$

or

$$
x^{\prime \prime \prime}(1)=0, x^{\prime \prime}(1)=0, x^{\prime}(1)=0, x(0)=\sum_{i=1}^{m-2} \beta_{i} x\left(\xi_{i}\right)
$$

where $0<\xi_{1}<\xi_{2}<\ldots<\xi_{m-2}<1, \beta_{i}>0,1=1,2, \ldots, m-2, \sum_{i=1}^{m-2} \beta_{i}>1$, and $f \in C([0,1] \times$ $\left.R^{4},[0,+\infty)\right)$.

One can see that all lower order derivatives are involved in the nonlinear term explicitly and the BCs are the m-point cases. In this sense, the problems studied in this paper are more general than before. In the paper, multiple monotone and convex positive solutions for problems (1.1), (1.2) and (1.1), (1.3) are established. The results presented extend the study for fourth-order boundary value problems of nonlinear ordinary differential equations.

This paper is organized as follows. In Section 2, we present some preliminaries and lemmas. Section 3 is devoted to the existence of at least three convex and increasing positive solutions for problem (1.1), (1.2). In Section 4, we prove that there exist at least three convex and decreasing positive solutions for problem (1.1), (1.3).

\section{Preliminaries and lemmas}

In this section, some preliminaries and lemmas used later are presented.

Definition 2.1 The map $\alpha$ is said to be a nonnegative continuous convex functional on cone $P$ of a real Banach space $E$ provided that $\alpha: P \rightarrow[0,+\infty)$ is continuous and

$$
\alpha(t x+(1-t) y) \leq t \alpha(x)+(1-t) \alpha(y) \quad \text { for all } x, y \in P \text { and } t \in[0,1] .
$$

Definition 2.2 The map $\beta$ is said to be a nonnegative continuous concave functional on cone $P$ of a real Banach space $E$ provided that $\beta: P \rightarrow[0,+\infty)$ is continuous and

$$
\beta(t x+(1-t) y) \geq t \beta(x)+(1-t) \beta(y), \quad \text { for all } x, y \in P \text { and } t \in[0,1] .
$$

Let $\gamma, \theta$ be nonnegative continuous convex functionals on $P, \alpha$ be a nonnegative continuous concave functional on $P$ and $\psi$ be a nonnegative continuous functional on $P$. Then for positive numbers $a, b, c$ and $d$, we define the following convex sets:

$$
\begin{aligned}
& P(\gamma, d)=\{x \in P \mid \gamma(x)<d\}, \\
& P(\gamma, \alpha, b, d)=\{x \in P \mid b \leq \alpha(x), \gamma(x) \leq d\}, \\
& P(\gamma, \theta, \alpha, b, c, d)=\{x \in P \mid b \leq \alpha(x), \theta(x) \leq c, \gamma(x) \leq d\}
\end{aligned}
$$


and a closed set

$$
R(\gamma, \psi, a, d)=\{x \in P \mid a \leq \psi(x), \gamma(x) \leq d\} .
$$

Lemma 2.1 [25] Let $P$ be a cone in Banach space $E$. Let $\gamma, \theta$ be nonnegative continuous convex functionals on $P, \alpha$ be a nonnegative continuous concave functional and $\psi$ be a nonnegative continuous functional on $P$ satisfying

$$
\psi(\lambda x) \leq \lambda \psi(x), \quad \text { for } 0 \leq \lambda \leq 1,
$$

such that for some positive numbers $l$ and $d$,

$$
\alpha(x) \leq \psi(x), \quad\|x\| \leq l \gamma(x)
$$

for all $x \in \overline{P(\gamma, d)}$. Suppose $T: \overline{P(\gamma, d)} \rightarrow \overline{P(\gamma, d)}$ is completely continuous and there exist positive numbers $a, b, c$ with $a<b$ such that

$\left(S_{1}\right)\{x \in P(\gamma, \theta, \alpha, b, c, d) \mid \alpha(x)>b\} \neq \varnothing$ and $\alpha(T x)>b$ for $x \in P(\gamma, \theta, \alpha, b, c, d)$;

$\left(S_{2}\right) \alpha(T x)>b$ for $x \in P(\gamma, \alpha, b, d)$ with $\theta(T x)>c$;

$\left(S_{3}\right) 0 \notin R(\gamma, \psi, a, d)$ and $\psi(T x)<a$ for $x \in R(\gamma, \psi, a, d)$ with $\psi(x)=a$.

Then $T$ has at least three fixed points $x_{1}, x_{2}, x_{3} \in \overline{P(\gamma, d)}$ such that:

$$
\gamma\left(x_{i}\right) \leq d, i=1,2,3 ; b<\alpha\left(x_{1}\right) ; a<\psi\left(x_{2}\right), \alpha\left(x_{2}\right)<b ; \psi\left(x_{3}\right)<a .
$$

\section{Positive solutions for problem (1.1), (1.2)}

We begin with the fourth-order $m$-point boundary value problem

$$
\begin{aligned}
& x^{(4)}(t)=y(t), t \in[0,1], \\
& x^{\prime \prime \prime}(1)=0, x^{\prime \prime}(1)=0, x^{\prime}(0)=0, \quad x(1)=\sum_{i=1}^{m-2} \beta_{i} x\left(\xi_{i}\right),
\end{aligned}
$$

where $0<\xi_{1}<\xi_{2}<\ldots<\xi_{m-2}<1, \beta_{i}>0, i=1,2, \ldots, m-2$.

The following assumption will stand throughout this section:

$$
\left(H_{1}\right) f \in C\left([0,1] \times R^{4},[0,+\infty)\right), \sum_{i=1}^{m-2} \beta_{i}>1, \sum_{i=1}^{m-2} \beta_{i} \xi_{i}<1 .
$$

Lemma 3.1 Denote $\xi_{0}=0, \xi_{m-1}=1, \beta_{0}=\beta_{m-1}=0$, and $y(t) \in C[0,1]$. Problem (3.1), (3.2) has the unique solution

$$
x(t)=\int_{0}^{1} G(t, s) y(s) d s,
$$

where

$$
G(t, s)= \begin{cases}-\frac{1}{6} t^{3}+\frac{1}{2} s t^{2}+\frac{1}{6} s^{3}+\frac{\sum_{k=0}^{i-1} \beta_{k}\left(\frac{1}{6} \xi_{k}^{3}-\frac{1}{2} \xi_{k}^{2} s-\frac{1}{6} s^{3}\right)+\frac{1}{2}\left(1-\sum_{k=i}^{m-2} \beta_{k} \xi_{k}\right) s^{2}}{\sum_{k=0}^{m-1} \beta_{k}-1}, t \leq s, \\ \frac{1}{2} s^{2} t+\frac{\sum_{k=0}^{i-1} \beta_{k}\left(\frac{1}{6} \xi_{k}^{3}-\frac{1}{2} \xi_{k}^{2} s-\frac{1}{6} s^{3}\right)+\frac{1}{2}\left(1-\sum_{k=i}^{m-2} \beta_{k} \xi_{k}\right) s^{2}}{\sum_{k=0}^{m-1} \beta_{k}-1}, & t \geq s,\end{cases}
$$


for $\xi_{i-1} \leq s \leq \xi_{i}, i=1,2, \ldots, m-1$.

Proof Let $G(t, s)$ be the Green's function of problem $x^{(4)}(t)=0$ with boundary condition (3.2). We can suppose

$$
G(t, s)=\left\{\begin{array}{l}
a_{3} t^{3}+a_{2} t^{2}+a_{1} t+a_{0}, \quad t \leq s, \xi_{i-1} \leq s \leq \xi_{i}, i=1,2, \ldots, m-1 \\
b_{3} t^{3}+b_{2} t^{2}+b_{1} t+b_{0}, \quad t \geq s, \xi_{i-1} \leq s \leq \xi_{i}, i=1,2, \ldots, m-1
\end{array}\right.
$$

Considering the definition and properties of Green's function together with the boundary condition (3.2), we have

$$
\left\{\begin{array}{l}
a_{3} s^{3}+a_{2} s^{2}+a_{1} s+a_{0}=b_{3} s^{3}+b_{2} s^{2}+b_{1} s+b_{0} \\
3 a_{3} s^{2}+2 a_{2} s+a_{1}=3 b_{3} s^{2}+2 b_{2} s+b_{1} \\
6 a_{3} s+2 a_{2}=6 b_{3} s+2 b_{2} \\
6 a_{3}-6 b_{3}=-1 \\
6 b_{3}=0,6 b_{3}+2 b_{2}=0 \\
a_{1}=0 \\
b_{3}+b_{2}+b_{1}+b_{0}=\sum_{k=0}^{i=1} \beta_{k}\left(a_{3} \xi_{k}^{3}+a_{2} \xi_{k}^{2}+a_{1} \xi_{k}+a_{0}\right)+\sum_{k=i}^{m-2} \beta_{k}\left(b_{3} \xi_{k}^{3}+b_{2} \xi_{k}^{2}+b_{1} \xi_{k}+b_{0}\right) .
\end{array}\right.
$$

A straightforward calculation shows that

$$
\begin{aligned}
& a_{3}=-\frac{1}{6}, a_{2}=\frac{s}{2}, a_{1}=0, a_{0}=\frac{\sum_{k=0}^{i-1} \beta_{k}\left(\frac{1}{6} \xi_{k}^{3}-\frac{1}{2} \xi_{k}^{2} s-\frac{1}{6} s^{3}\right)+\frac{1}{2}\left(1-\sum_{k=i}^{m-2} \beta_{k} \xi_{k}\right) s^{2}}{\sum_{k=0}^{m-1} \beta_{k}-1}+\frac{1}{6} s^{3}, \\
& b_{3}=b_{2}=0, b_{1}=\frac{s^{2}}{2}, b_{0}=\frac{\sum_{k=0}^{i-1} \beta_{k}\left(\frac{1}{6} \xi_{k}^{3}-\frac{1}{2} \xi_{k}^{2} s-\frac{1}{6} s^{3}\right)+\frac{1}{2}\left(1-\sum_{k=i}^{m-2} \beta_{k} \xi_{k}\right) s^{2}}{\sum_{k=0}^{m-1} \beta_{k}-1}
\end{aligned}
$$

These give the explicit expression of the Green's function and the proof of Lemma 3.1 is completed.

Lemma 3.2 One can see that $G(t, s) \geq 0, t, s \in[0,1]$.

Proof For $\xi_{i-1} \leq s \leq \xi_{i}, i=1,2, \ldots, m-1$,

$$
\frac{\partial G(t, s)}{\partial t}= \begin{cases}\frac{1}{2} t(2 s-t), & t \leq s, \xi_{i-1} \leq s \leq \xi_{i} \\ \frac{1}{2} s^{2}, & t \geq s, \quad \xi_{i-1} \leq s \leq \xi_{i}\end{cases}
$$

Then $\frac{\partial G(t, s)}{\partial t} \geq 0,0 \leq t, s \leq 1$. Thus $G(t, s)$ is increasing on $t$. By a simple computation, we see

$$
G(0, s)=\frac{1}{6} s^{3}+\frac{\sum_{k=0}^{i-1} \beta_{k}\left(\frac{1}{6} \xi_{k}^{3}-\frac{1}{2} \xi_{k}^{2} s-\frac{1}{6} s^{3}\right)+\frac{1}{2}\left(1-\sum_{k=i}^{m-2} \beta_{k} \xi_{k}\right) s^{2}}{\sum_{k=0}^{m-1} \beta_{k}-1} \geq 0 .
$$

These ensures that $G(t, s) \geq 0, t, s \in[0,1]$.

Lemma 3.3 Suppose $x(t) \in C^{3}[0,1]$ and

$$
x^{\prime \prime \prime}(1)=0, x^{\prime \prime}(1)=0, x^{\prime}(0)=0, x(1)=\sum_{i=1}^{m-2} \beta_{i} x\left(\xi_{i}\right)
$$


Furthermore $x^{(4)}(t) \geq 0$ and there exist $t_{0}$ such that $x^{(4)}\left(t_{0}\right)>0$. Then $x(t)$ has the following properties:

(1) $\min _{0 \leq t \leq 1}|x(t)| \geq \delta \max _{0 \leq t \leq 1}|x(t)|$,

(2) $\max _{0 \leq t \leq 1}|x(t)| \leq \gamma \max _{0 \leq t \leq 1}\left|x^{\prime}(t)\right|$,

(3) $\max _{0 \leq t \leq 1}\left|x^{\prime}(t)\right| \leq \max _{0 \leq t \leq 1}\left|x^{\prime \prime}(t)\right| \max _{0 \leq t \leq 1}\left|x^{\prime \prime}(t)\right| \leq \max _{0 \leq t \leq 1}\left|x^{\prime \prime \prime}(t)\right|$,

where $\delta=\left(1-\sum_{i=1}^{m-2} \beta_{i} \xi_{i}\right) / \sum_{i=1}^{m-2} \beta_{i}\left(1-\xi_{i}\right), \gamma=\sum_{i=1}^{m-2} \beta_{i}\left(1-\xi_{i}\right) /\left(\sum_{i=1}^{m-2} \beta_{i}-1\right)$ are positive constants.

Proof Since $x^{(4)}(t) \geq 0, t \in[0,1]$, then $x^{\prime \prime \prime}(t)$ is increasing on $[0,1]$. Considering $x^{\prime \prime \prime}(1)=$ 0 , we have $x^{\prime \prime}(t) \leq 0, t \in[0,1]$. Thus $x^{\prime \prime}(t)$ is decreasing on $[0,1]$. Considering this together with the boundary condition $x^{\prime \prime}(1)=0$, we conclude that $x^{\prime \prime}(t) \geq 0$. Then $x(t)$ is convex on $[0,1]$. Taking into account that $x^{\prime}(0)=0$, we get that

$$
\max _{0 \leq t \leq 1} x(t)=x(1), \min _{0 \leq t \leq 1} x(t)=x(0)
$$

(1) From the concavity of $x(t)$, we have

$$
\xi_{i}(x(1)-x(0)) \geq x\left(\xi_{i}\right)-x(0) .
$$

Multiplying both sides with $\beta_{i}$ and considering the boundary condition, we have

$$
\left(1-\sum_{i=1}^{m-2} \beta_{i} \xi_{i}\right) x(1) \leq \sum_{i=1}^{m-2} \beta_{i}\left(1-\xi_{i}\right) x(0) .
$$

Thus

$$
\min _{0 \leq t \leq 1}|x(t)| \geq \delta \max _{0 \leq t \leq 1}|x(t)| .
$$

(2) Considering the mean-value theorem together with the concavity of $x(t)$, we have

$$
x(1)-x\left(\xi_{i}\right) \leq\left(1-\xi_{i}\right) x^{\prime}(1) .
$$

Multiplying both sides with $\beta_{i}$ and considering the boundary condition, we have

$$
\left(\sum_{i=1}^{m-2} \beta_{i}-1\right) x(1) \leq \sum_{i=1}^{m-2} \beta_{i}\left(1-\xi_{i}\right) x^{\prime}(1),
$$

which yields that $x(1) \leq \sum_{i=1}^{m-2} \beta_{i}\left(1-\xi_{i}\right) /\left(\sum_{i=1}^{m-2} \beta_{i}-1\right)\left|x^{\prime}(1)\right|=\gamma \max _{0 \leq t \leq 1}\left|x^{\prime}(t)\right|$.

(3) For $x^{\prime}(t)=x^{\prime}(0)+\int_{0}^{t} x^{\prime \prime}(s) d s$ and $x^{\prime}(0)=0$, we get

$$
\left|x^{\prime}(t)\right|=\left|\int_{0}^{t} x^{\prime \prime}(s) d s\right| \leq \int_{0}^{1}\left|x^{\prime \prime}(s)\right| d s .
$$

For $x^{\prime \prime}(t)=x^{\prime \prime}(1)-\int_{t}^{1} x^{\prime \prime \prime}(s) d s$ and $x^{\prime \prime}(1)=0$, we get

$$
\left|x^{\prime \prime}(t)\right|=\left|\int_{t}^{1} x^{\prime \prime \prime}(s) d s\right| \leq \int_{0}^{1}\left|x^{\prime \prime \prime}(s)\right| d s .
$$

\section{Consequently}

$$
\max _{0 \leq t \leq 1}\left|x^{\prime}(t)\right| \leq \max _{0 \leq t \leq 1}\left|x^{\prime \prime}(t)\right|, \max _{0 \leq t \leq 1}\left|x^{\prime \prime}(t)\right| \leq \max _{0 \leq t \leq 1}\left|x^{\prime \prime \prime}(t)\right| .
$$


These give the proof of Lemma 3.3.

Remark Lemma 3.3 ensures that

$$
\max \left\{\max _{0 \leq t \leq 1}|x(t)|, \max _{0 \leq t \leq 1}\left|x^{\prime}(t)\right|, \max _{0 \leq t \leq 1}\left|x^{\prime \prime}(t)\right|, \max _{0 \leq t \leq 1}\left|x^{\prime \prime \prime}(t)\right|\right\} \leq \gamma \max _{0 \leq t \leq 1}\left|x^{\prime \prime \prime}(t)\right| .
$$

Let Banach space $E=C^{3}[0,1]$ be endowed with the norm

$$
\|x\|=\max \left\{\max _{0 \leq t \leq 1}|x(t)|, \max _{0 \leq t \leq 1}\left|x^{\prime}(t)\right|, \max _{0 \leq t \leq 1}\left|x^{\prime \prime}(t)\right|, \max _{0 \leq t \leq 1}\left|x^{\prime \prime \prime}(t)\right|\right\}, x \in E .
$$

Define the cone $P \subset E$ by

$$
P=\left\{x \in E \mid x(t) \geq 0, x^{\prime \prime \prime}(1)=0, x^{\prime \prime}(1)=0, x^{\prime}(0)=0, x(1)=\sum_{i=1}^{m-2} \beta_{i} x\left(\xi_{i}\right), x(t) \text { is convex on }[0,1]\right\} .
$$

Let the nonnegative continuous concave functional $\alpha$, the nonnegative continuous convex functionals $\gamma, \theta$ and the nonnegative continuous functional $\psi$ be defined on the cone by

$$
\gamma(x)=\max _{0 \leq t \leq 1}\left|x^{\prime \prime \prime}(t)\right|, \theta(x)=\psi(x)=\max _{0 \leq t \leq 1}|x(t)|, \alpha(x)=\min _{0 \leq t \leq 1}|x(t)| .
$$

By Lemma 3.3, the functionals defined above satisfy

$$
\delta \theta(x) \leq \alpha(x) \leq \theta(x)=\psi(x),\|x\| \leq \gamma \gamma(x) .
$$

Denote

$$
m=\int_{0}^{1} G(0, s) d s, N=\int_{0}^{1} G(1, s) d s, \lambda=\min \{m, \delta \gamma\} .
$$

Assume that there exist constants $0<a, b, d$ with $a<b<\lambda d$ such that

$\left(A_{1}\right) f(t, u, v, w, p) \leq d, \quad(t, u, v, w, p) \in[0,1] \times[0, \gamma d] \times[0, d] \times[0, d] \times[-d, 0]$,

$\left(A_{2}\right) f(t, u, v, w, p)>b / m,(t, u, v, w, p) \in[0,1] \times[b, b / \delta] \times[0, d] \times[0, d] \times[-d, 0]$,

$\left(A_{3}\right) f(t, u, v, w, p)<a / N,(t, u, v, w, p) \in[0,1] \times[0, a] \times[0, d] \times[0, d] \times[-d, 0]$.

Theorem 3.1 Under assumptions $\left(A_{1}\right)-\left(A_{3}\right)$, problem (1.1), (1.2) has at least three positive solutions $x_{1}, x_{2}, x_{3}$ satisfying

$$
\begin{aligned}
& \max _{0 \leq t \leq 1}\left|x^{\prime \prime \prime}{ }_{i}(t)\right| \leq d, i=1,2,3 ; b<\min _{0 \leq t \leq 1}\left|x_{1}(t)\right| ; \\
& a<\max _{0 \leq t \leq 1}\left|x_{2}(t)\right|, \min _{0 \leq t \leq 1}\left|x_{2}(t)\right|<b ; \\
& \quad \max _{0 \leq t \leq 1}\left|x_{3}(t)\right| \leq a .
\end{aligned}
$$

Proof Problem $(1.1,1.2)$ has a solution $x=x(t)$ if and only if $x$ solves the operator equation

$$
x(t)=\int_{0}^{1} G(t, s) f\left(s, x(s), x^{\prime}(s), x^{\prime \prime}(s), x^{\prime \prime \prime}(s)\right) d s=(T x)(t) .
$$

Then

$$
(T x)^{\prime \prime \prime}(t)=-\int_{t}^{1} f\left(s, x, x^{\prime}, x^{\prime \prime}, x^{\prime \prime \prime}\right) d s .
$$

For $x \in \overline{P(\gamma, d)}$, considering Lemma 3.3 and assumption $\left(A_{1}\right)$, we have $f\left(t, x(t), x^{\prime}(t)\right.$, $\left.x^{\prime \prime}(t), x^{\prime \prime \prime}(t)\right) \leq d$. Thus 


$$
\gamma(T x)=\left|(T x)^{\prime \prime \prime}(0)\right|=\left|-\int_{0}^{1} f\left(s, x, x^{\prime}, x^{\prime \prime}, x^{\prime \prime \prime}\right) d s\right|=\int_{0}^{1}\left|f\left(s, x, x^{\prime}, x^{\prime \prime}, x^{\prime \prime \prime}\right)\right| d s \leq d .
$$

Hence $T: \overline{P(\gamma, d)} \rightarrow \overline{P(\gamma, d)}$. An application of the Arzela-Ascoli theorem yields that $T$ is a completely continuous operator. The fact that the constant function $x(t)=b / \delta$ $\in P(\gamma, \theta, \alpha, b, c, d)$ and $\alpha(b / \delta)>b$ implies that

$$
\{x \in P(\gamma, \theta, \alpha, b, c, d) \mid \alpha(x)>b\} \neq \emptyset .
$$

For $x \in P(\gamma, \theta, \alpha, b, c, d)$, we have $b \leq x(t) \leq b / \delta$ and $\left|x^{\prime \prime \prime}(t)\right|<d$. From assumption $\left(A_{2}\right)$, we see

$$
f\left(t, x, x^{\prime}, x^{\prime \prime}, x^{\prime \prime \prime}\right)>b / m \text {. }
$$

Hence, by definition of $\alpha$ and the cone $P$, we can get

$$
\alpha(T x)=(T x)(0)=\int_{0}^{1} G(0, s) f\left(s, x, x^{\prime}, x^{\prime \prime}, x^{\prime \prime \prime}\right) d s \geq \frac{b}{m} \int_{0}^{1} G(0, s) d s>\frac{b}{m} m=b,
$$

which means $\alpha(T x)>b, \forall x \in P(\gamma, \theta, \alpha, b, b / \delta, d)$. This ensures that condition (S1) of Lemma 2.1 is fulfilled.

Second, with (3.4) and $b<\lambda d$, we have

$$
\alpha(T x) \geq \delta \theta(T x)>\delta \times \frac{b}{\delta}=b
$$

for all $x \in P(\gamma, \alpha, b, d)$ with $\theta(T x)>\frac{b}{\delta}$.

Thus, condition $\left(S_{2}\right)$ of Lemma 2.1 holds. Finally we show that $\left(S_{3}\right)$ also holds. We see $\psi(0)=0<a$ and $0 \notin R(\gamma, \psi, a, d)$. Suppose that $x \in R(\gamma, \psi, a, d)$ with $\psi(x)=a$, then by the assumption of $\left(A_{3}\right)$,

$$
\psi(T x)=\max _{0 \leq t \leq 1}|(T x)(t)|=\int_{0}^{1} G(1, s) f\left(s, x, x^{\prime}, x^{\prime \prime}, x^{\prime \prime \prime}\right) d s<\frac{a}{N} \int_{0}^{1} G(1, s) d s=a,
$$

which ensures that condition $\left(S_{3}\right)$ of Lemma 2.1 is fulfilled. Thus, an application of Lemma 2.1 implies that the fourth-order m-point boundary value problem $(1.1,1.2)$ has at least three positive convex increasing solutions $x_{1}, x_{2}, x_{3}$ with the properties that

$$
\begin{gathered}
\max _{0 \leq t \leq 1}\left|x_{i}^{\prime \prime \prime}(t)\right| \leq d, i=1,2,3 ; b<\min _{0 \leq t \leq 1}\left|x_{1}(t)\right| ; \\
a<\max _{0 \leq t \leq 1}\left|x_{2}(t)\right|, \min _{0 \leq t \leq 1}\left|x_{2}(t)\right|<b ; \\
\max _{0 \leq t \leq 1}\left|x_{3}(t)\right| \leq a .
\end{gathered}
$$

\section{Positive solutions for problem (1.1), (1.3)}

The following assumption will stand throughout this section:

$$
\left(H_{2}\right), f \in C\left([0,1] \times R^{4},[0,+\infty)\right), \sum_{i=1}^{m-2} \beta_{i}>1, \sum_{i=1}^{m-2} \beta_{i} \xi_{i}+1-\sum_{i=1}^{m-2} \beta_{i}>0 .
$$

Lemma 4.1 Denote $\xi_{0}=0, \xi_{m-1}=1, \beta_{0}=\beta_{m-1}=0$, the Green's function of problem

$$
x^{(4)}(t)=0,
$$




$$
x^{\prime \prime \prime}(1)=0, x^{\prime \prime}(1)=0, x^{\prime}(1)=0, x(0)=\sum_{i=1}^{m-2} \beta_{i} x\left(\xi_{i}\right)
$$

is

$$
H(t, s)= \begin{cases}-\frac{1}{6} t^{3}+\frac{1}{2} s t^{2}-\frac{1}{2} s^{2} t+\frac{\sum_{k=0}^{i-1} \beta_{k}\left(\frac{1}{6} \xi_{k}^{3}-\frac{1}{2} \xi_{k}^{2} s+\frac{1}{2} \xi_{k} s^{2}\right)+\sum_{k=i}^{m-2} \frac{1}{6} \beta_{k} s^{3}}{\sum_{k=0}^{m-1} \beta_{k}-1}, t \leq s, \quad \xi_{i-1} \leq s \leq \xi_{i}, \\ -\frac{1}{6} s^{3}+\frac{\sum_{k=0}^{i-1} \beta_{k}\left(\frac{1}{6} \xi_{k}^{3}-\frac{1}{2} \xi_{k}^{2} s+\frac{1}{2} \xi_{k} s^{2}\right)+\sum_{k=i}^{m-2} \frac{1}{6} \beta_{k} s^{3}}{\sum_{k=0}^{m-1} \beta_{k}-1}, & t \geq s, \xi_{i-1} \leq s \leq \xi_{i},\end{cases}
$$

for $i=1,2, \ldots, m-1$.

Proof Suppose that

$$
H(t, s)=\left\{\begin{array}{l}
a_{3} t^{3}+a_{2} t^{2}+a_{1} t+a_{0} t \leq s, \xi_{i-1} \leq s \leq \xi_{i}, i=1,2, \ldots, m-1 \\
b_{3} t^{3}+b_{2} t^{2}+b_{1} t+b_{0} t \geq s, \xi_{i-1} \leq s \leq \xi_{i}, i=1,2, \ldots, m-1
\end{array}\right.
$$

Considering the definition and properties of Green's function together with the boundary condition (4.2), we have

$$
\left\{\begin{array}{l}
a_{3} s^{3}+a_{2} s^{2}+a_{1} s+a_{0}=b_{3} s^{3}+b_{2} s^{2}+b_{1} s+b_{0} \\
3 a_{3} s^{2}+2 a_{2} s+a_{1}=3 b_{3} s^{2}+2 b_{2} s+b_{1} \\
6 a_{3} s+2 a_{2}=6 b_{3} s+2 b_{2} \\
6 a_{3}-6 b_{3}=-1 \\
b_{3}=0 \\
6 b_{3}+2 b_{2}=0 \\
3 b_{3}+2 b_{2}+b_{1}=0 \\
a_{0}=\sum_{k=0}^{i=1} \beta_{k}\left(a_{3} \xi_{k}^{3}+a_{2} \xi_{k}^{2}+a_{1} \xi_{k}+a_{0}\right)+\sum_{k=i}^{m-2} \beta_{k}\left(b_{3} \xi_{k}^{3}+b_{2} \xi_{k}^{2}+b_{1} \xi_{k}+b_{0}\right) .
\end{array}\right.
$$

Consequently

$$
\begin{aligned}
& a_{3}=-\frac{1}{6}, a_{2}=\frac{1}{2} s, a_{1}=-\frac{1}{2} s^{2}, b_{3}=b_{2}=b_{1}=0, \\
& a_{0}=\frac{\sum_{k=0}^{i-1} \beta_{k}\left(\frac{1}{6} \xi_{k}^{3}-\frac{1}{2} \xi_{k}^{2} s+\frac{1}{2} \xi_{k} s^{2}\right)+\sum_{k=i}^{m-2} \frac{1}{6} \beta_{k} s^{3}}{\sum_{k=0}^{m-1} \beta_{k}-1}, \\
& b_{0}=\frac{\sum_{k=0}^{i-1} \beta_{k}\left(\frac{1}{6} \xi_{k}^{3}-\frac{1}{2} \xi_{k}^{2} s+\frac{1}{2} \xi_{k} s^{2}\right)+\sum_{k=i}^{m-2} \frac{1}{6} \beta_{k} s^{3}}{\sum_{k=0}^{m-1} \beta_{k}-1}-\frac{1}{6} s^{3} .
\end{aligned}
$$

The proof of Lemma 4.1 is completed.

Lemma 4.2 One can see that $H(t, s) \geq 0, t, s \in[0,1]$.

Proof For $\xi_{i-1} \leq s \leq \xi_{i}, i=1,2, \ldots, m-1$,

$$
\frac{\partial H(t, s)}{\partial t}= \begin{cases}-\frac{1}{2}(t-s)^{2}, & t \leq s, \xi_{i-1} \leq s \leq \xi_{i} \\ 0, & t \geq s, \quad \xi_{i-1} \leq s \leq \xi_{i} .\end{cases}
$$


Then $\frac{\partial H(t, s)}{\partial t} \leq 0,0 \leq t, s \leq 1$, which implies that $H(t, s)$ is decreasing on $t$. The fact that

$$
H(1, s)=\frac{\sum_{k=0}^{i-1} \beta_{k}\left(\frac{1}{6} \xi_{k}^{3}-\frac{1}{2} \xi_{k}^{2} s+\frac{1}{2} \xi_{k} s^{2}\right)+\sum_{k=i}^{m-2} \frac{1}{6} \beta_{k} s^{3}}{\sum_{k=0}^{m-1} \beta_{k}-1}-\frac{1}{6} s^{3} \geq 0
$$

ensures that $H(t, s) \geq 0, t, s \in[0,1]$.

Lemma 4.3 If $x(t) \in C^{3}[0,1]$,

$$
x^{\prime \prime \prime}(1)=0, x^{\prime \prime}(1)=0, x^{\prime}(1)=0, x(0)=\sum_{i=1}^{m-2} \beta_{i} x\left(\xi_{i}\right)
$$

and $x^{(4)}(t) \geq 0$, there exists $t_{0}$ such that $x^{(4)}\left(t_{0}\right)>0$, then

(1) $\min _{0 \leq t \leq 1}|x(t)| \geq \delta_{1} \max _{0 \leq t \leq 1}|x(t)|$,

(2) $\max _{0 \leq t \leq 1}|x(t)| \leq \gamma_{1} \max _{0 \leq t \leq 1}\left|x^{\prime}(t)\right|$,

(3) $\max _{0 \leq t \leq 1}\left|x^{\prime}(t)\right| \leq \max _{0 \leq t \leq 1}\left|x^{\prime \prime}(t)\right|, \max _{0 \leq t \leq 1}\left|x^{\prime \prime}(t)\right| \leq \max _{0 \leq t \leq 1}\left|x^{\prime \prime \prime}(t)\right|$

where $\delta_{1}=\left(1-\sum_{i=1}^{m-2} \beta_{i}\left(1-\xi_{i}\right)\right) / \sum_{i=1}^{m-2} \beta_{i} \xi_{i}, \gamma_{1}=\sum_{i=1}^{m-2} \beta_{i} \xi_{i} /\left(\sum_{i=1}^{m-2} \beta_{i}-1\right)$ are positive constants.

Proof It follows from the same methods as Lemma 3.3 that $x(t)$ is convex on $[0,1]$. Taking into account that $x^{\prime}(1)=0$, one can see that $x(t)$ is decreasing on $[0,1]$ and

$$
\max _{0 \leq t \leq 1} x(t)=x(0), \min _{0 \leq t \leq 1} x(t)=x(1) .
$$

(1) From the concavity of $x(t)$, we have

$$
\xi_{i}(x(1)-x(0)) \geq x\left(\xi_{i}\right)-x(0) .
$$

Multiplying both sides with $\beta_{i}$ and considering the boundary condition, we have

$$
\sum_{i=1}^{m-2} \beta_{i} \xi_{i} x(1) \geq\left(1-\sum_{i=1}^{m-2} \beta_{i}\left(1-\xi_{i}\right)\right) x(0) .
$$

Thus

$$
\min _{0 \leq t \leq 1}|x(t)| \geq \delta_{1} \max _{0 \leq t \leq 1}|x(t)| .
$$

(2) Considering the mean-value theorem, we get

$$
(0)-x\left(\xi_{i}\right) \leq \xi_{i}\left|x^{\prime}(0)\right| \text {. }
$$

From the concavity of $x$ similarly with above we know

$$
\left(\sum_{i=1}^{m-2} \beta_{i}-1\right) x(0)<\sum_{i=1}^{m-2} \beta_{i} \xi_{i}\left|x^{\prime}(0)\right| .
$$

Considering (4.3) together with (4.4) we have $x(0) \leq \gamma_{1}\left|x^{\prime}(0)\right|=\gamma_{1} \max _{0 \leq t \leq 1}\left|x^{\prime}(t)\right|$.

(3) For $x^{\prime}(t)=x^{\prime}(1)-\int_{t}^{1} x^{\prime \prime}(s) d s, x^{\prime \prime}(t)=x^{\prime \prime}(1)-\int_{t}^{1} x^{\prime \prime \prime}(s) d s$ and $x^{\prime}(1)=0, x^{\prime \prime}(1)=0$, we get 


$$
\left|x^{\prime}(t)\right|=\left|\int_{t}^{1} x^{\prime \prime}(s) d s\right| \leq \int_{0}^{1}\left|x^{\prime \prime}(s)\right| d s,\left|x^{\prime \prime}(t)\right|=\left|\int_{t}^{1} x^{\prime \prime \prime}(s) d s\right| \leq \int_{0}^{1}\left|x^{\prime \prime \prime}(s)\right| d s .
$$

Thus

$$
\max _{0 \leq t \leq 1}\left|x^{\prime}(t)\right| \leq \max _{0 \leq t \leq 1}\left|x^{\prime \prime}(t)\right|, \max _{0 \leq t \leq 1}\left|x^{\prime \prime}(t)\right| \leq \max _{0 \leq t \leq 1}\left|x^{\prime \prime \prime}(t)\right| .
$$

Remark We see that

$$
\max \left\{\max _{0 \leq t \leq 1}|x(t)|, \max _{0 \leq t \leq 1}\left|x^{\prime}(t)\right|, \max _{0 \leq t \leq 1}\left|x^{\prime \prime}(t)\right|, \max _{0 \leq t \leq 1}\left|x^{\prime \prime \prime}(t)\right|\right\} \leq \gamma_{1} \max _{0 \leq t \leq 1}\left|x^{\prime \prime \prime}(t)\right| .
$$

Let Banach space $E=C^{3}[0,1]$ be endowed with the norm

$$
\|x\|=\max \left\{\max _{0 \leq t \leq 1}|x(t)|, \max _{0 \leq t \leq 1}\left|x^{\prime}(t)\right|, \max _{0 \leq t \leq 1}\left|x^{\prime \prime}(t)\right|, \max _{0 \leq t \leq 1}\left|x^{\prime \prime \prime}(t)\right|\right\}, x \in E .
$$

Define the cone $P \subset E$ by

$$
P_{1}=\left\{x \in E \mid x(t) \geq 0, x^{\prime \prime \prime}(1)=0, x^{\prime \prime}(1)=0, x^{\prime}(1)=0, x(0)=\sum_{i=1}^{m-2} \beta_{i} x\left(\xi_{i}\right), x(t) \text { is convex on }[0,1]\right\} .
$$

Denote

$$
m_{1}=\int_{0}^{1} H(1, s) d s, N_{1}=\int_{0}^{1} H(0, s) d s, \lambda_{1}=\min \left\{m_{1}, \delta_{1} \gamma_{1}\right\}
$$

Assume that there exist constants $0<a, b, d$ with $a<b<\lambda_{1} d$ such that

$$
\begin{aligned}
& \left(A_{4}\right) f(t, u, v, w, p) \leq d, \quad(t, u, v, w, p) \in[0,1] \times\left[0, v_{1} d\right] \times[-d, 0] \times[0, d] \times[-d, 0], \\
& \left(A_{5}\right) f(t, u, v, w, p)>b / m_{1},(t, u, v, w, p) \in[0,1] \times\left[b, b / \delta_{1}\right] \times[-d, 0] \times[0, d] \times[-d, 0], \\
& \left(A_{6}\right) f(t, u, v, w, p)<a / N_{1},(t, u, v, w, p) \in[0,1] \times[0, a] \times[-d, 0] \times[0, d] \times[-d, 0] .
\end{aligned}
$$

Theorem 4.1 Under assumptions $\left(A_{4}\right)-\left(A_{6}\right)$, problem (1.1), (1.3) has at least three positive solutions $x_{1}, x_{2}, x_{3}$ with the properties that

$$
\max _{0 \leq t \leq 1}\left|x_{i}^{\prime \prime \prime}(t)\right| \leq d, i=1,2,3 ; b<\min _{0 \leq t \leq 1}\left|x_{1}(t)\right| ; a<\max _{0 \leq t \leq 1}\left|x_{2}(t)\right|, \min _{0 \leq t \leq 1}\left|x_{2}(t)\right|<b ; \max _{0 \leq t \leq 1}\left|x_{3}(t)\right| \leq a .
$$

Proof Problem (1.1), (1.3) has a solution $x=x(t)$ if and only if $x$ solves the operator equation

$$
x(t)=\int_{0}^{1} H(t, s) f\left(s, x(s), x^{\prime}(s), x^{\prime \prime}(s), x^{\prime \prime \prime}(s)\right) d s=\left(T_{1} x\right)(t) .
$$

Then

$$
\left(T_{1} x\right)^{\prime \prime \prime}(t)=-\int_{t}^{1} f\left(s, x, x^{\prime}, x^{\prime \prime}, x^{\prime \prime \prime}\right) d s .
$$

For $x \in \overline{P_{1}(\gamma, d)}$, considering Lemma 4.3 and assumption $\left(A_{4}\right)$, we have

$$
f\left(t, x(t), x^{\prime}(t), x^{\prime \prime}(t), x^{\prime \prime \prime}(t)\right) \leq d .
$$

Thus

$$
\gamma\left(T_{1} x\right)=\left|\left(T_{1} x\right)^{\prime \prime \prime}(0)\right|=\left|-\int_{0}^{1} f\left(s, x, x^{\prime}, x^{\prime \prime}, x^{\prime \prime \prime}\right) d s\right|=\int_{0}^{1}\left|f\left(s, x, x^{\prime}, x^{\prime \prime}, x^{\prime \prime \prime}\right)\right| d s \leq d .
$$

Hence $T_{1}: \overline{P_{1}(\gamma, d)} \rightarrow \overline{P_{1}(\gamma, d)}$ and $T_{1}$ is a completely continuous operator obviously. The fact 
that the constant function $x(t)=b / \delta_{1} \in P_{1}(\gamma, \theta, \alpha, b, c, d)$ and $\alpha\left(b / \delta_{1}\right)>b$ implies that

$$
\left\{x \in P_{1}(\gamma, \theta, \alpha, b, c, d \mid \alpha(x)>b)\right\} \neq \emptyset .
$$

This ensures that condition (S1) of Lemma 2.1 holds.

For $x \in P_{1}(\gamma, \theta, \alpha, b, c, d)$, we have $b \leq x(t) \leq b / \delta_{1}$ and $\left|x^{\prime \prime \prime}(t)\right|<d$. From assumption $\left(A_{4}\right)$,

$$
f\left(t, x, x^{\prime}, x^{\prime \prime}, x^{\prime \prime \prime}\right)>b / m_{1} .
$$

Hence, by definition of $\alpha$ and the cone $P_{1}$, we can get

$$
\alpha\left(T_{1} x\right)=\left(T_{1} x\right)(1)=\int_{0}^{1} H(1, s) f\left(s, x, x^{\prime}, x^{\prime \prime}, x^{\prime \prime \prime}\right) d s \geq \frac{b}{m_{1}} \int_{0}^{1} H(1, s) d s>\frac{b}{m_{1}} m_{1}=b,
$$

which means $\alpha\left(T_{1} x\right)>b, \forall x \in P_{1}(\gamma, \theta, \alpha, b, b / \delta, d)$.

Second, with (4.4) and $b<\lambda_{1} d$, we have

$$
\alpha\left(T_{1} x\right) \geq \delta_{1} \theta\left(T_{1} x\right)>\delta_{1} \times \frac{b}{\delta_{1}}=b
$$

for all $x \in P_{1}(\gamma, \alpha, b, d)$ with $\theta\left(T_{1} x\right)>\frac{b}{\delta_{1}}$.

Thus, condition $\left(S_{2}\right)$ of Lemma 2.1 holds. Finally we show that $\left(S_{3}\right)$ also holds. We see $\psi(0)=0<a$ and $0 \notin R(\gamma, \psi, a, d)$. Suppose that $x \in R(\gamma, \psi, a, d)$ with $\psi(x)=a$, then by the assumption of $\left(A_{6}\right)$,

$$
\psi\left(T_{1} x\right)=\max _{0 \leq t \leq 1}\left|\left(T_{1} x\right)(t)\right|=\int_{0}^{1} H(0, s) f\left(s, x, x^{\prime}, x^{\prime \prime}, x^{\prime \prime \prime}\right) d s<\frac{a}{N_{1}} \int_{0}^{1} H(0, s) d s=a,
$$

which ensures that condition $\left(S_{3}\right)$ of Lemma 2.1 is satisfied. Thus, an application of Lemma 2.1 implies that the fourth-order m-point boundary value problem (1.1), (1.3) has at least three positive convex decreasing solutions $x_{1}, x_{2}, x_{3}$ satisfying the conditions that

$$
\max _{0 \leq t \leq 1}\left|x_{i}^{\prime \prime \prime}(t)\right| \leq d, i=1,2,3 ; b<\min _{0 \leq t \leq 1}\left|x_{1}(t)\right| ; a<\max _{0 \leq t \leq 1}\left|x_{2}(t)\right|, \min _{0 \leq t \leq 1}\left|x_{2}(t)\right|<b ; \max _{0 \leq t \leq 1}\left|x_{3}(t)\right| \leq a .
$$

\section{Acknowledgements}

We are indebted to the anonymous referee for a detailed reading and useful comments and suggestions, which allowed us to improve this work. This work was supported by the Anhui Provincial Natural Science Foundation (10040606Q50), National Natural Science Foundation of China (No.11071164), Shanghai Natural Science Foundation (No.10ZR1420800)

\section{Author details}

${ }^{1}$ College of Science, University of Shanghai for Science and Technology, Shanghai 200093, PR China ${ }^{2}$ Department of Mathematics, Hefei Normal University, Hefei, Anhui Province 230061, PR China

\section{Authors' contributions}

The authors declare that the work was realized in collaboration with the same responsibility. All authors read and approved the final manuscript.

\section{Competing interests}

The authors declare that they have no competing interests.

Received: 27 December 2010 Accepted: 5 September 2011 Published: 5 September 2011

\section{References}

1. Ma, R: Positive solutions of a nonlinear three-point boundary valve problem. Eur J Differ Equ. 34, 1-8 (1999) 
2. Ma, R, Cataneda, N: Existence of solution for nonlinear m-point boundary value problem. J Math Anal Appl. 256, 556-567 (2001). doi:10.1006/jmaa.2000.7320

3. Ma, R, Wang, H: Positive solutions of nonlinear three-point boundary value problems. J Math Anal Appl. 279, 1216-1227 (2003)

4. He, X, Ge, W: Triple solutions for second-order three-point boundary value problems. J Math Anal Appl. 268, 256-265 (2002). doi:10.1006/jmaa.2001.7824

5. Guo, Y, Ge, W: Positive solutions for three-point boundary-value problems with dependence on the first order derivative. J Math Anal Appl. 290, 291-301 (2004). doi:10.1016/j.jmaa.2003.09.061

6. Avery, Rl., et al: Twin solutions of boundary value problems for ordinary differential equations and finite difference equations. Comput Math Appl. 42, 695-704 (2001). doi:10.1016/50898-1221(01)00188-2

7. Avery, Rl, Henderson, J: Three symmetric positive solutions for a second order boundary-value problem. Appl Math Lett. $13,1-7(2000)$

8. Henderson, J: Double solutions of three-point boundary-value problems for second-order differential equations. Electron J Differ Equ. 115, 1-7 (2004)

9. Eloe, PW, Henderson, J: Positive solutions for $(n-1, n)$ conjugate boundary value problems. Nonlinear Anal. 28 1669-1680 (1997). doi:10.1016/0362-546X(95)00238-0

10. Yang, L., et al: Multiplicity results for second-order m-point boundary value problem. J Math Anal Appl. 324, 532-542 (2006). doi:10.1016/j.jmaa.2005.07.084

11. Webb, JRL, Infante, G: Positive solutions of nonlocal boundary value problems: a unified approach. J Lond Math Soc. 74, 673-693 (2006). doi:10.1112/S0024610706023179

12. Webb, JRL, Infante, G: Positive solutions of nonlocal boundary value problems involving integral conditions. Nonlinear Differ Equ Appl. 15, 45-67 (2008). doi:10.1007/s00030-007-4067-7

13. Agarwal, RP, O'Regan, D: A multiplicity result for second order impulsive differential equations via the Leggett Williams fixed point theorem. Appl Math Comput. 161, 433-439 (2005). doi:10.1016/j.amc.2003.12.096

14. Agarwal, RP: Focal Boundary Value Problems for Differential and Difference Equations. Kluwer Academic, Dordrecht (1998)

15. Agarwal, RP, O'Regan, D, Wong, PJY: Positive Solutions of Differential, Difference and Integral Equations. Kluwer Academic, Dordercht (1998)

16. Yao, Q: Positive solutions for Eigenvalue problems of fourth-order elastic beam equation. Appl Math Lett. 17, 237-243 (2004). doi:10.1016/50893-9659(04)90037-7

17. $L i, Y$ : On the existence of positive solutions for the bending elastic beam equations. Appl Math Comput. 189, 821-827 (2007). doi:10.1016/j.amc.2006.11.144

18. Yang, Y, Zhang, J: Nontrival solutions for some fourth-order boundary value problems with parametes. Nonlinear Anal. 70, 3966-3977 (2009). doi:10.1016/j.na.2008.08.005

19. Yang, Y: Triple positive solutions of a class of fourth-order two-point boundary value problems. Appl Math Lett. 23, 366-370 (2010). doi:10.1016/j.aml.2009.10.012

20. Yang, X, Lo, K: Existence of a positive solution of a fourth-order boundary value problems. Nonlinear Anal. 69 , 2267-2273 (2008). doi:10.1016/j.na.2007.08.006

21. Hang, G, Xu, Z: Multiple solutions of some fourth-order beam equations. Nonlinear Anal. 68, 3646-3656 (2008). doi:10.1016/j.na.2007.04.007

22. Liu, Y: Multiple positive solutions of nonlinear singular boundary value problem for fourth-order equations. Appl Math Lett. 17, 747-757 (2004). doi:10.1016/j.aml.2004.06.001

23. Bai, Z, Wang, H: On positive solutions of some fourth-order beam equations. J Math Anal Appl. 270, 357-368 (2002). doi:10.1016/50022-247X(02)00071-9

24. Kaufmann, RR, Kosmatov, N: Elastic beam equation with high order derivatives. Nonlinear Anal Real World Appl. 8, 811-821 (2007). doi:10.1016/j.nonrwa.2006.03.006

25. Avery, Rl, Peterson, AC: Three positive fixed points of nonlinear operators on an ordered Banach space. Comput Math Appl. 208, 313-322 (2001)

doi:10.1186/1687-2770-2011-21

Cite this article as: Liu et al:: Monotone and convex positive solutions for fourth-order multi-point boundary value problems. Boundary Value Problems 2011 2011:21.

\section{Submit your manuscript to a SpringerOpen ${ }^{\circ}$ journal and benefit from:}

Convenient online submission

- Rigorous peer review

- Immediate publication on acceptance

- Open access: articles freely available online

- High visibility within the field

- Retaining the copyright to your article

Submit your next manuscript at $\gg$ springeropen.com 